ARTICLE OPEN

\title{
PRMT5 regulates cell pyroptosis by silencing CASP1 in multiple myeloma
}

\author{
Tian Xia (iD) ${ }^{1}$, Ming Liu², Quan Zhao ${ }^{2}$, Jian Ouyang (iD ${ }^{1 凶}$, Peipei Xu (D) ${ }^{1,3^{凶}}$ and Bing Chen (iD ${ }^{\boxplus}$ \\ (c) The Author(s) 2021, corrected publication 2021
}

Protein arginine methyltransferase 5 (PRMT5), a histone methyltransferase responsible for the symmetric dimethylation of histone $\mathrm{H} 4$ on $\mathrm{Arg} 3$ (H4R3me2s), is an enzyme that participates in tumor cell progression in a variety of hematological malignancies. However, the biological functions of PRMT5 in multiple myeloma (MM) and the underlying molecular mechanisms remain unclear. In this study, we conducted a bioinformatics analysis and found that PRMT5 expression was significantly upregulated in MM. In vitro and in vivo phenotypic experiments revealed that knockdown of PRMT5 expression enhanced cell pyroptosis in MM. Moreover, we found that CASP1 expression was negatively correlated with PRMT5 expression, and repressing PRMT5 expression rescued both the phenotype and expression markers (N-GSDMD, IL-1b, and IL-18). Inhibition of PRMT5 activity increased CASP1 expression and promoted MM cell pyroptosis. Finally, high expression of PRMT5 or low expression of CASP1 was correlated with poor overall survival in MM. Collectively, our results provide a mechanism by which PRMT5 regulates cell pyroptosis by silencing CASP1 in MM.

Cell Death and Disease (2021)12:851; https://doi.org/10.1038/s41419-021-04125-5

\section{INTRODUCTION}

Multiple myeloma (MM) is a malignancy that originates from the extensive proliferation of pathological antibody-producing plasma cells in the bone marrow and accounts for $\sim 10 \%$ of hematological cancers [1]. From 2006 to 2016, 16,500 new cases of MM were reported in China, and the age-standardized mortality rate (ASMR) was $0.67 / 100,000$ in 2016 [2]. Many patients with MM suffer from bone destruction and hypercalcemia as a result of osteoclast activation and osteoblast inhibition caused by cytokines expressed by MM cells [3], and from renal failure as a result of the accumulation and precipitation of light chains produced by high amounts of $\mathrm{MM}$ cells [4]. At present, many studies have proven that individuals with MM share the same genetic features; thus, MM is thought to arise as a result of the accumulation of genetic alterations. Hypermethylation of many tumor suppressor genes, such as SOCS-1 $[5,6]$ and RUNX2 [7], has been shown to contribute to MM progression [8]. Nevertheless, the development of $\mathrm{MM}$ is a complicated pathological process that requires further study.

Protein arginine methyltransferase 5 (PRMT5) is the main type II methyltransferase that catalyzes the symmetric addition of dimethylarginine to histone proteins, nonhistone proteins, and cytoplasmic proteins and is involved in numerous cellular processes, including transcription, DNA repair, RNA processing, proliferation, and metabolism [9-11]. Several studies have demonstrated that PRMT5 is overexpressed in hematological malignancies, including leukemia, lymphoma, and MM [12]. Gullà et al. [13] have found that the PRMT5 inhibitor EPZ015666 induces cell autophagy via the PRMT5/TRIM21/IKKB axis, which is identifying PRMT5 as a promising therapeutic target in MM. However, there is still a lack of detailed evidence to explain how PRMT5 triggers the proliferation of MM cells in detail.

In this study, in addition to our cohort, we extracted clinical and RNA sequencing data from the Multiple Myeloma Research Foundation (MMRF) CoMMpass study to assess the clinical significance of PRMT5. We also explored the biological function of PRMT5 in MM cells and investigated the mechanism by which PRMT5 regulates MM cell pyroptosis by repressing CASP1.

\section{RESULTS}

\section{PRMT5 was overexpressed in MM}

A bioinformatics analysis was performed on seven MM datasets from the Oncomine database. Consequently, PRMT5 was found to be significantly upregulated in $M M$ tissues compared to noncancerous tissues $(P=0.020$, Fig. 1A). In addition, further bioinformatics analysis combining the MMRF CoMMpass $(n=$ $859)$ and Genotype-Tissue Expression (GTEx) databases $(n=62)$ was conducted, which revealed that PRMT5 expression was higher in $\mathrm{MM}$ tissues than in noncancerous tissues ( $P=0.00034$, Fig. 1B). Moreover, by analyzing the clinical data from 787 patients with MM from the MMRF CoMMpass database, we found that high PRMT5 expression was associated with poor progression-free survival $(P=0.015, \mathrm{HR}=1.472$, Fig. $1 \mathrm{C})$. We determined the expression of PRMT5 mRNA in bone marrow-derived plasma cells from 35 MM samples, 17 MGUS samples, and 10 noncancerous samples using quantitative real-time polymerase chain reaction (qRT-PCR) analysis to verify the database results. PRMT5 levels

\footnotetext{
${ }^{1}$ Department of Hematology, The Affiliated Drum Tower Hospital of Nanjing University Medical School, Nanjing 210008 Jiangsu, People's Republic of China. ${ }^{2}$ The State Key Laboratory of Pharmaceutical Biotechnology, Nanjing University, Nanjing 210008, People's Republic of China. ${ }^{3}$ Clinical College of Traditional Chinese and Western Medicine, Nanjing University of Chinese Medicine, Nanjing 210008 Jiangsu, People's Republic of China. ${ }^{\bowtie}$ email: ouyangj211@163.com; xu_peipei0618@163.com; chenb211@163.com Edited by Professor Stephen Tait
}

Received: 24 January 2021 Revised: 30 July 2021 Accepted: 23 August 2021

Published online: 16 September 2021 
A

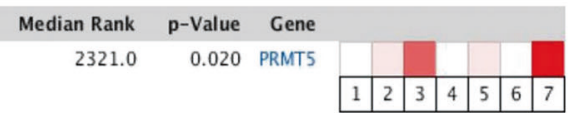

$\begin{array}{llllllll}1 & 5 & 10 & 25 & 25 & 10 & 5 & 1\end{array}$ $\square \square \square \square \square \square \square \square \square \square$ Not measured

B

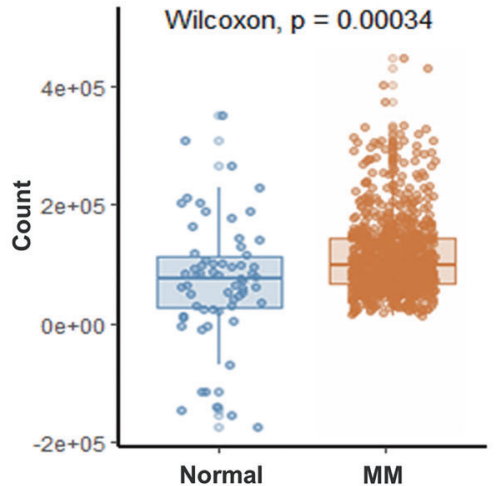

C

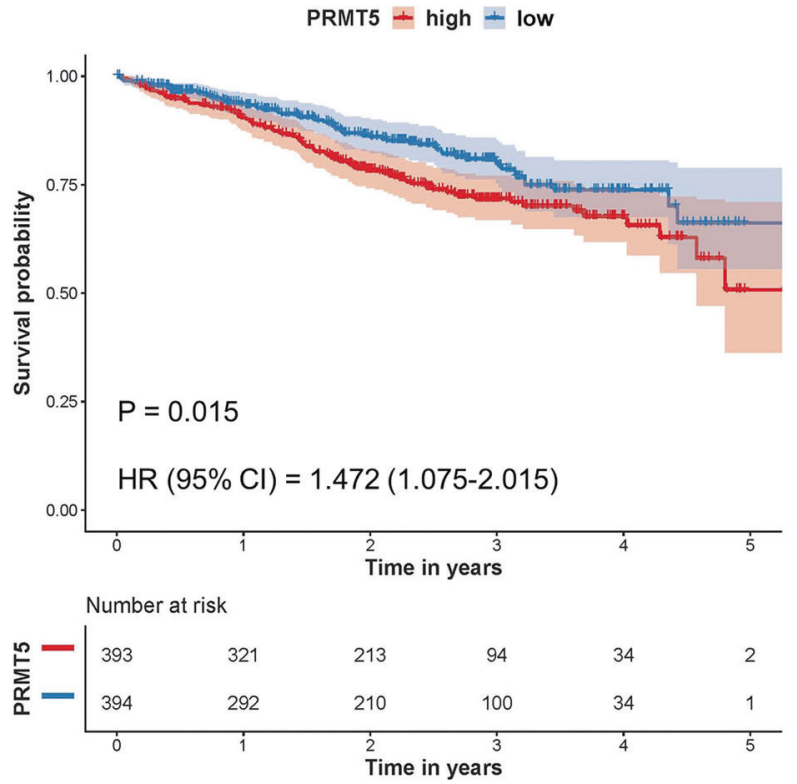

D

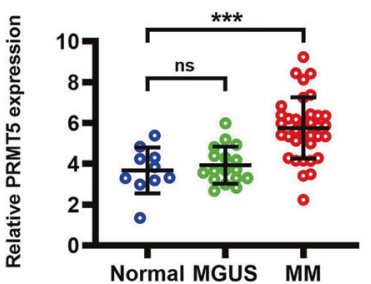

E

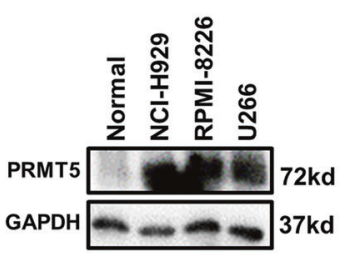

F

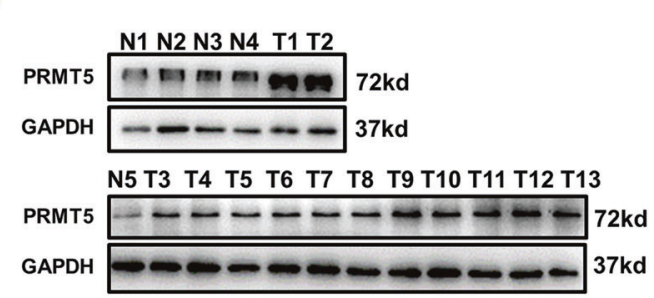

Fig. 1 PRMT5 is overexpressed in MM. A PRMT5 expression in 7 MM files from the Oncomine. B Relative expression of PRMT5 in MM tissues $(n=859)$ and noncancerous tissues $(n=62)$ from the MMRF CoMMpass and GTEx databases. C Progression-free survival times in MM patients with low versus high expression of PRMT5 assessed by Kaplan-Meier analysis from the MMRF CoMMpass cohorts. D Relative mRNA expression of PRMT5 in MM $(n=35)$ and MGUS $(n=17)$ tissues compared with that in noncancerous $(n=10)$ tissues via qRT-PCR. E PRMT5 protein level in $13 \mathrm{MM}$ tissues and 5 normal tissues via western blotting. $\mathbf{F}$ PRMT5 protein level in normal plasma cells and $3 \mathrm{MM}$ cell lines (NCI-H929, RPMI-8226, and U266) via western blotting. Data shown are mean \pm SD. ${ }^{* * *} P<0.001$.

were significantly higher in MM samples than in control samples, while no significant change was observed in MGUS samples (Fig. 1D). Also, datasets including MGUS samples (GSE6477 and GSE5900) showed no difference in PRMT5 expression between healthy donors and MGUS patients. In addition, by using western blotting assays, the PRMT5 protein was found to be overexpressed in $M M$ samples and MM cell lines (Fig. 1E, F).

\section{Knockdown of PRMT5 triggered cell membrane rupture and elevated $\mathrm{PI}+$ /Annexin $\mathrm{V}+$ population in MM cells}

When investigating the function of PRMT5 in MM cells, we designed two specific short hairpin RNAs (shRNAs) and successfully knocked down the relative levels of PRMT5 expression in two MM cell lines (NCl-H929 and U266) compared to that in the negative control group as assessed by western blotting and qRTPCR (Fig. 2A, B).These results were further confirmed by immunofluorescence-targeting PRMT5 in both cell lines (Fig. 2C).

Cell Counting Kit-8 (CCK-8) assays indicated that downregulation of PRMT5 promoted cell viability in the two individual MM cell lines $(P<0.05$, Supplemental Fig. 1A). In addition, knockdown of PRMT5 was confirmed to increase the $\mathrm{PI}+$ population of $\mathrm{NCl}-\mathrm{H} 929$ and U266 cells by flow cytometry, particularly in the PI+/Annexin $\mathrm{V}+$ domain (Fig. 2D). This phenotype could refer to late apoptosis or pyroptosis, both of which manifest as the coloration of PI dye caused by the poles of cell membrane. Since the activation of caspase 3 (CASP3) is a trigger of the execution pathway in apoptosis, we analyzed the expression of cleaved-CASP3 in shPRMT5 cells and negative control cells, but the results showed no significance (Fig. 2E). Furthermore, TEM images revealed shPRMT5 cells with cytoplasmic swelling and plasma membrane rupture, which supported the morphology of pyroptosis (Fig. 2F). These results suggested that knockdown of PRMT5 activates pyroptosis in MM cell lines.

\section{PRMT5 upregulation was inversely correlated with CASP1}

We conducted a genome array on three $\mathrm{NCl}-\mathrm{H} 929$ cell samples and three PRMT5-knockdown (PRMT5-kd) NCl-H929 cell samples using Affymetrix Clariom $\mathrm{S}$ array chips to determine the function of PRMT5 in MM pathogenesis (Fig. 3A). Datasets and profiles were uploaded to the Gene Expression Omnibus database (GSE162715). Relative gene expression changes of the top 15 significantly upregulated genes in the PRMT5-kd group were explored, including CASP1, PRG2, AHSP, GIMAP2, SAMD9, HEPACAM2, SLC4A1, SUCNR1, OAS2, FOSL2, LIPH, CCDC30, KLHL6, ATP10D, and NCEH1. Results revealed that CASP1 was significantly increased in both $\mathrm{NCl}-\mathrm{H} 929$ and $\mathrm{U} 266$ cells (Fig. 3B). To confirm that CASP1 acts as a downstream target of PRMT5, we evaluated protein expression levels of CASP1 and cleaved-CASP1 in MM cell lines after transfection with PRMT5-shRNA-2 compared to the negative control groups. Notably, knockdown of PRMT5 led to significant elevation of CASP1 and cleaved-CASP1, indicating that CASP1 was silenced by PRMT5 in MM cells (Fig. 3C). In addition, 
A

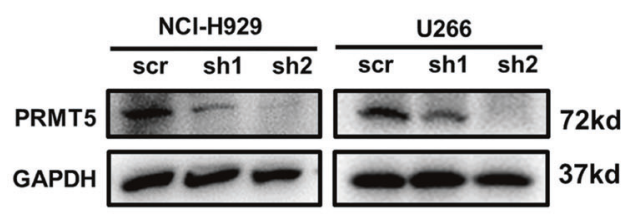

B
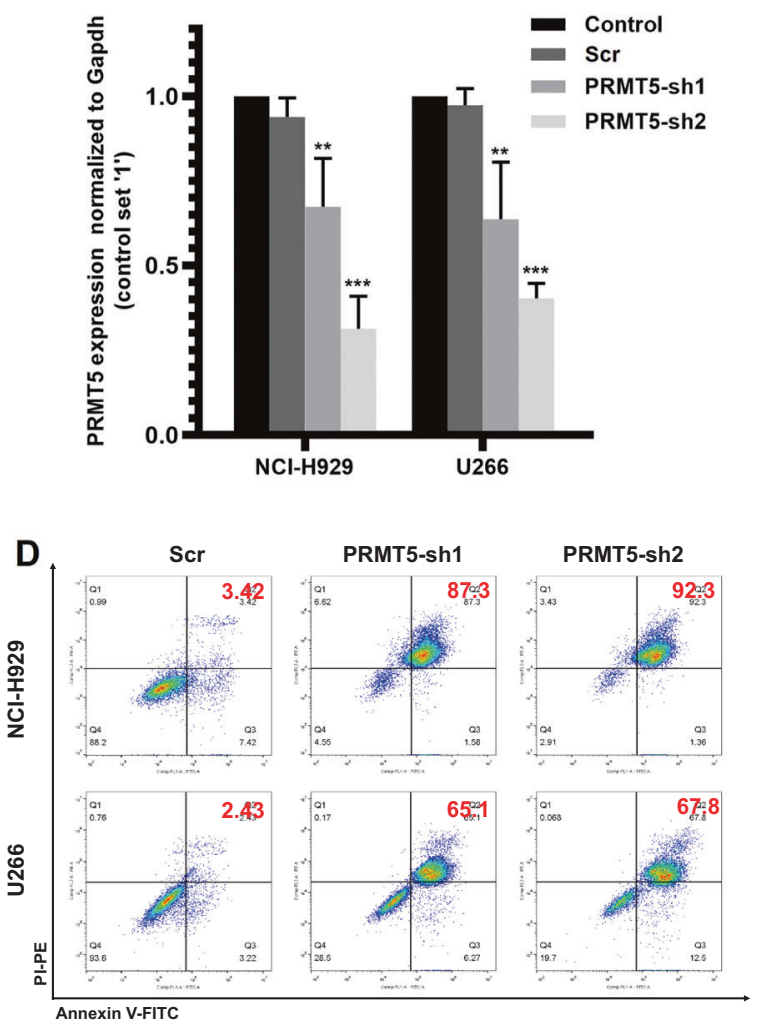

C
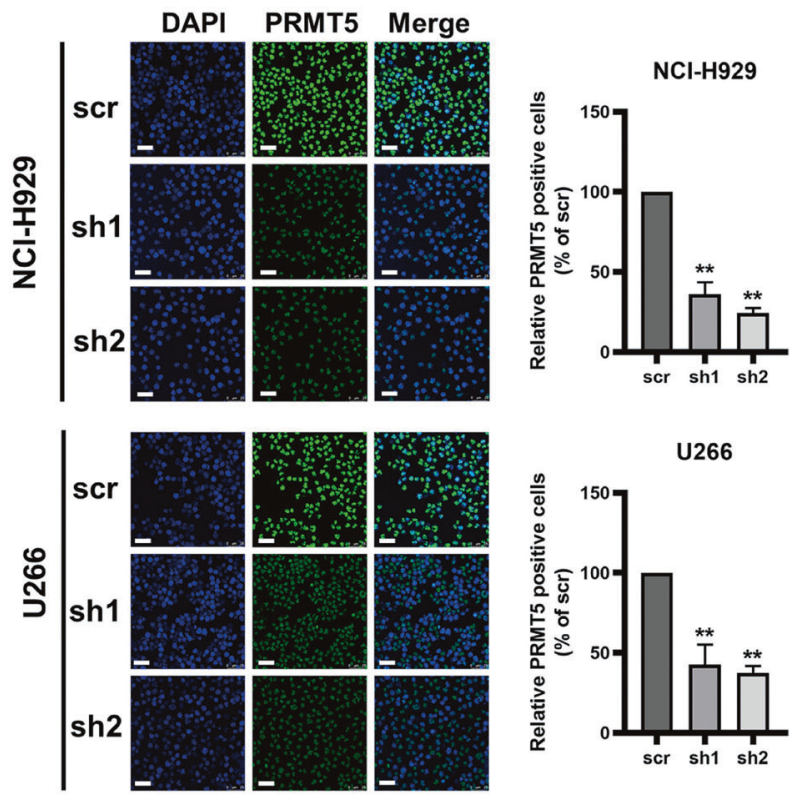

U266

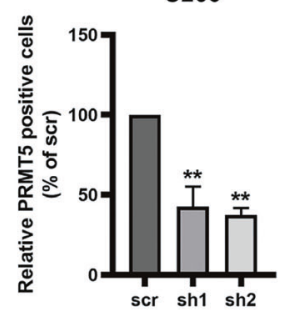

E

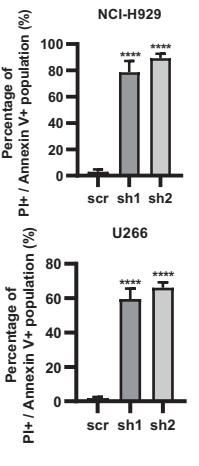

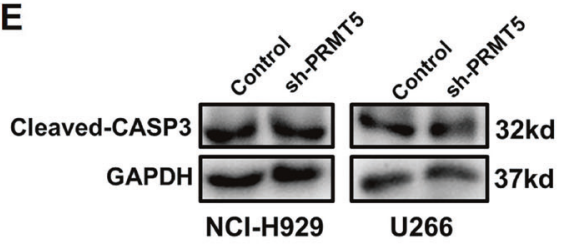

F

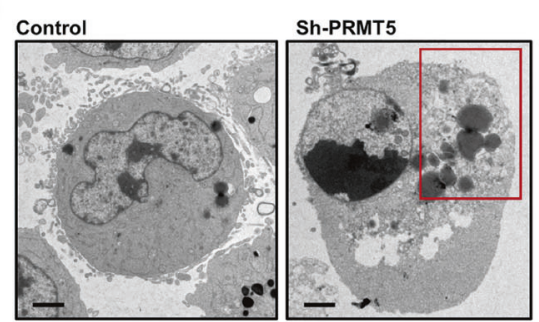

Fig. 2 PRMT5 activates pyroptosis of MM cell lines. A-C PRMT5 knockdown was detected in NCl-H929 and U266 cells stably expressing the scrambled control (scr) or PRMT5 shRNA (PRMT5 sh1/2) by western blotting, qRT-PCR, and immunofluorescence assay. Scale bar $=25 \mu$ m. D The analysis of Annexin V-FITC and PI by flow cytometry in NCl-H929 (upper panel) and U266 (lower panel) cells stably expressing the control or PRMT5 shRNA. E Expression level of cleaved-CASP3 in shPRMT5 cells and negative control cells via western blotting. F Representative images of electron microscopy analysis of pyroptosis morphology changes in NCl-H929 cells. Scale bar $=2 \mu \mathrm{m}$. Data shown are mean \pm SD $(n=3)$. ${ }^{* *} P<0.01,{ }^{* * *} P<0.001,{ }^{* * * *} P<0.0001$.

CASP1 mRNA expression in bone marrow-derived plasma cells was significantly decreased and negatively correlated with PRMT5 in patients with MM (Fig. 3D, E).

\section{Pyroptosis induced by depletion of PRMT5 was rescued by knockdown of CASP1 in MM cell lines}

To investigate whether PRMT5 regulates cell pyroptosis by repressing CASP1 expression, we transiently overexpressed CASP1 in $\mathrm{NCl}-\mathrm{H} 929$ and U266 cells (Fig. 4A). Our results revealed that CASP1 overexpression significantly suppressed cell viability (Supplemental Fig. 1B) and increased the population of $\mathrm{PI}$ + /Annexin V+ cells in NCl-H929 and U266 cells (Fig. 4B). Our results also showed that overexpression of CASP1 enhanced the activation of GSDMD and the secretion of interleukin-1 beta (IL-1b) and interleukin-18 (IL-18) (Fig. 4C). Similarly, CASP1 expression was elevated in PRMT5-depleted cells, which activated pyroptosis pathways. However, when CASP1 was knocked down by siRNAs in PRMT5-depleted cells (Fig. 4D), markers of the pyroptosis pathway were significantly downregulated, and the percentage of pyroptotic cells was remarkably decreased (Fig. 4E, F). Cell growth was rescued after depletion of CASP1 in shPRMT5 cells (Supplemental Fig. 1C). Furthermore, we performed in vivo experiments using mouse xenograft models and found that the knockdown of CASP1 partially reversed the proliferation defect in PRMT5depleted $\mathrm{NCl}-\mathrm{H} 929$ cells in vivo (Fig. 4G, H). The results described above demonstrated that cell pyroptosis induced by depletion of PRMT5 is rescued by knockdown of CASP1.

\section{Treatment with the PRMT5 inhibitor GSK591 reduced the level} of H4R3me2s at the CASP1 promoter

We designed six pairs of walking primers across the core promoter regions of the CASP1 gene to test whether PRMT5 directly regulates CASP1 expression and analyzed the enrichment of PRMT5-mediated H4R3me2s around the CASP1 gene promoter using chromatin immunoprecipitation (ChIP) assays and QRT-PCR. Our results demonstrated that, in PRMT5-depleted NCI-H929 and U266 cells, the levels 
A

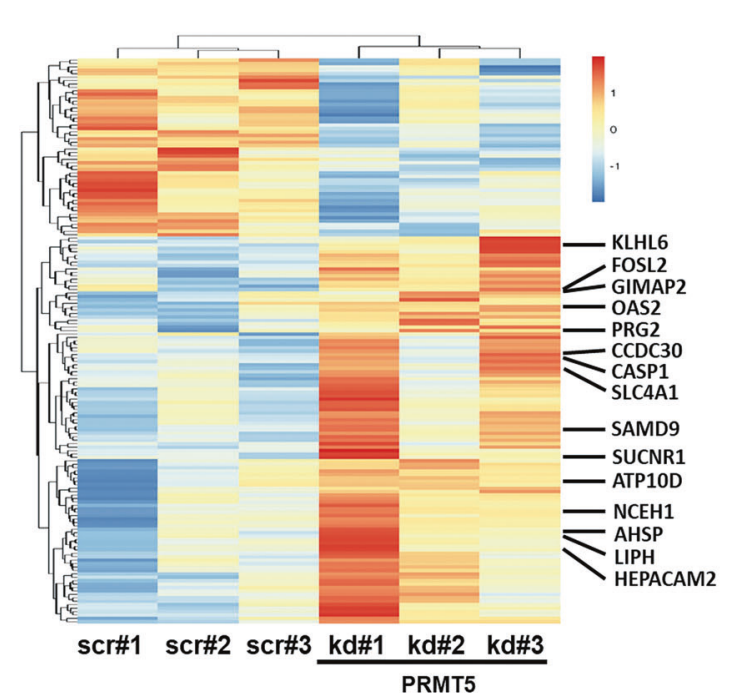

B

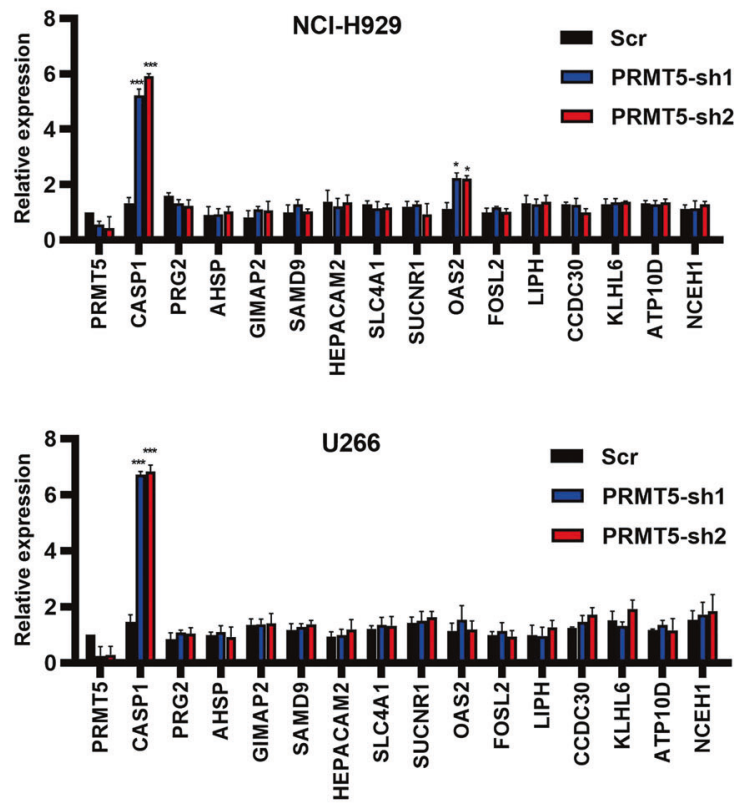

C

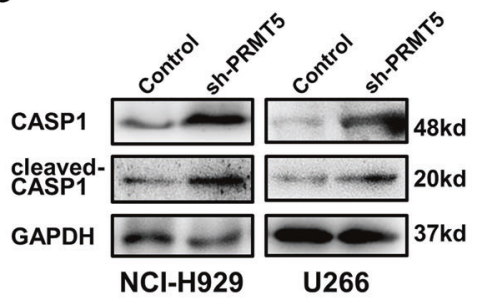

D

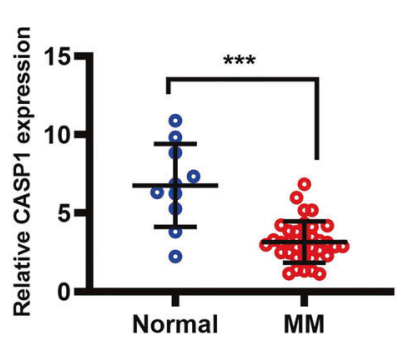

E

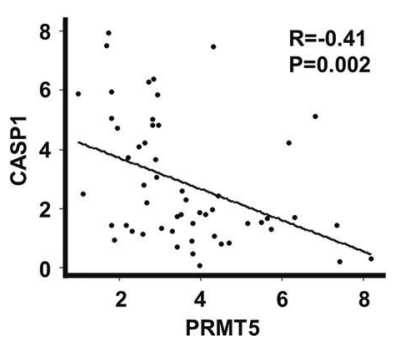

Fig. 3 PRMT5 upregulation is inversely correlated with CASP1. A Heatmap showing differentially expressed genes after PRMT5 knockdown. Highlighted genes are the top 15 significantly upregulated genes in NCl-H929 cells stably expressing PRMT5 shRNA. B Relative mRNA expression levels of significantly upregulated genes in NCl-H929 cells or U266 cells stably expressing PRMT5 shRNA compared to scr shRNA via qRT-PCR. GAPDH was used as an endogenous control. C Expression levels of CASP1 and cleaved-CASP1 were both upregulated in NCI-H929 cells or U266 cells stably expressing PRMT5 shRNA than scr shRNA via western blotting. D Relative mRNA expression of CASP1 was downregulated in MM $(n=35)$ tissues compared with that in noncancerous $(n=10)$ tissues by qRT-PCR. E The Pearson's correlation analysis of CASP1 and PRMT5 in bone marrow tissues of MM patients $(n=55)$ from the MMRF CoMMpass database. Data shown are mean \pm SD $(n=3)$. ${ }^{*} P<0.05,{ }^{* * *} P<0.001$.

of H4R3me2s were significantly reduced at the promoter region of CASP1 compared to the control group (Fig. 5A).

To inhibit H4R3me2s at the CASP1 gene promoter, we treated $\mathrm{NCl}-\mathrm{H} 929$ and U266 cells with GSK591 (also known as EPZ015866), which is widely acknowledged as a specific inhibitor of PRMT5 $[14,15]$. We treated $\mathrm{NCl}-\mathrm{H} 929$ and $\mathrm{U} 266$ cells with $0,1,5$ and $10 \mu \mathrm{M}$ GSK591 (B6182, Apexbio) to investigate its biological function. We found that $5 \mu \mathrm{M}$ GSK591 treatment significantly triggered an increase in the proportion of $\mathrm{PI}+$ cells in both $\mathrm{NCl}$ H929 and U266 cells, suggesting membrane rupture and secondary cell death caused by pyroptosis (Fig. 5B). Next, we examined the expression of CASP1 in NCl-H929 and U266 cells treated with various concentrations of GSK591 to explore whether inhibiting the enzymatic activity of PRMT5 could reactivate CASP1 expression. We found that $5 \mu \mathrm{M}$ GSK591 increased CASP1 expression (Fig. 5C). Our results indicated that GSK591 reduced the levels of H4R3me2s and accordingly activated CASP1mediated cell pyroptosis by inhibiting PRMT5.

High expression of PRMT5 and low expression of CASP1 were associated with poor clinical outcomes in patients with MM We collected clinical data from 108 newly diagnosed MM patients in our hospital from 2008 to 2020, and determined the PRMT5 and
CASP1 expression levels by immunohistochemistry. Bone marrow tissues from MM patients were divided into PRMT5-high and PRMT5-low groups (Fig. 6A), as well as CASP1-high and CASP1-low groups (Fig. 6B). A significant association between PRMT5 expression and ISS stage was observed in patients with MM (Table 1). Additionally, Kaplan-Meier curves (Fig. 6C, D) demonstrated that high PRMT5 expression $(P=0.022)$ and low CASP1 expression $(P=0.00063)$ were associated with poor overall survival in MM. We merged these two variants and found that patients with both low PRMT5 and high CASP1 expression exhibited significantly worse survival compared with to those carrying high PRMT5 and low CASP1 expression (Fig. 6E, $P=$ $0.0037)$. Moreover, both the univariate $(P<0.001)$ and multivariate $(P=0.007)$ Cox proportional hazards regression analyses demonstrated that PRMT5 upregulation was an independent prognostic risk factor for poor survival in patients with MM (Table 2).

\section{DISCUSSION}

In the present study, we observed that PRMT5 expression was significantly upregulated in MM bone marrow tissue, which might serve as an adverse prognostic factor for overall survival in patients with MM. Moreover, PRMT5 suppressed cell pyroptosis 
A

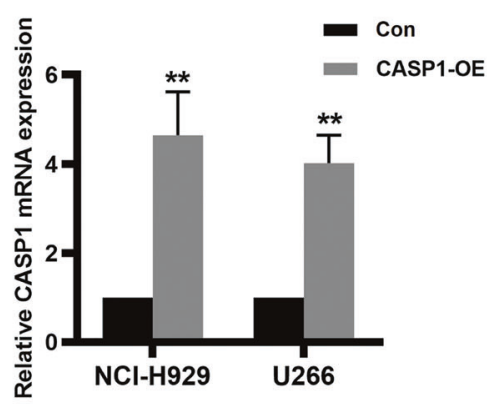

B
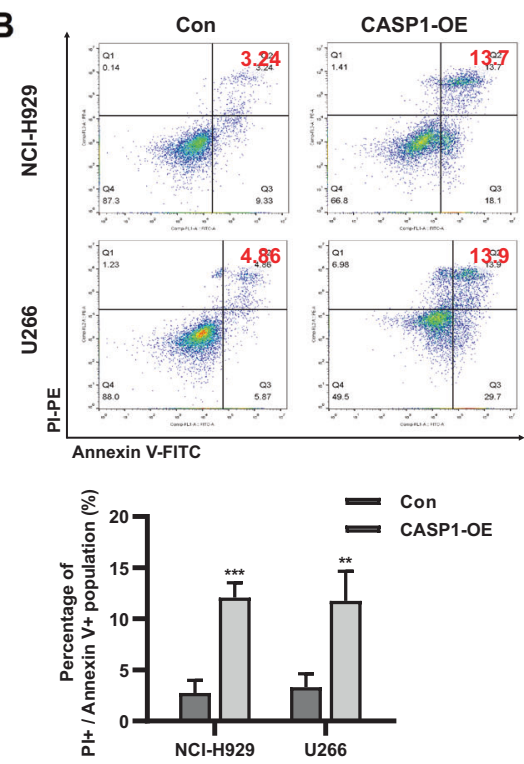

C

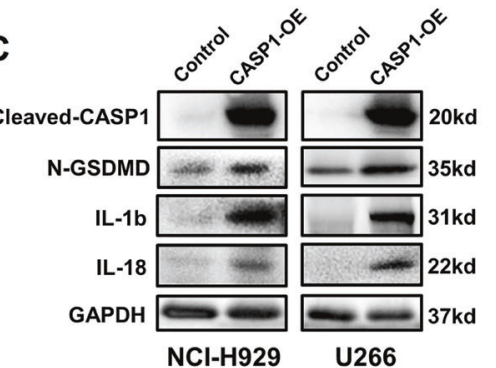

D
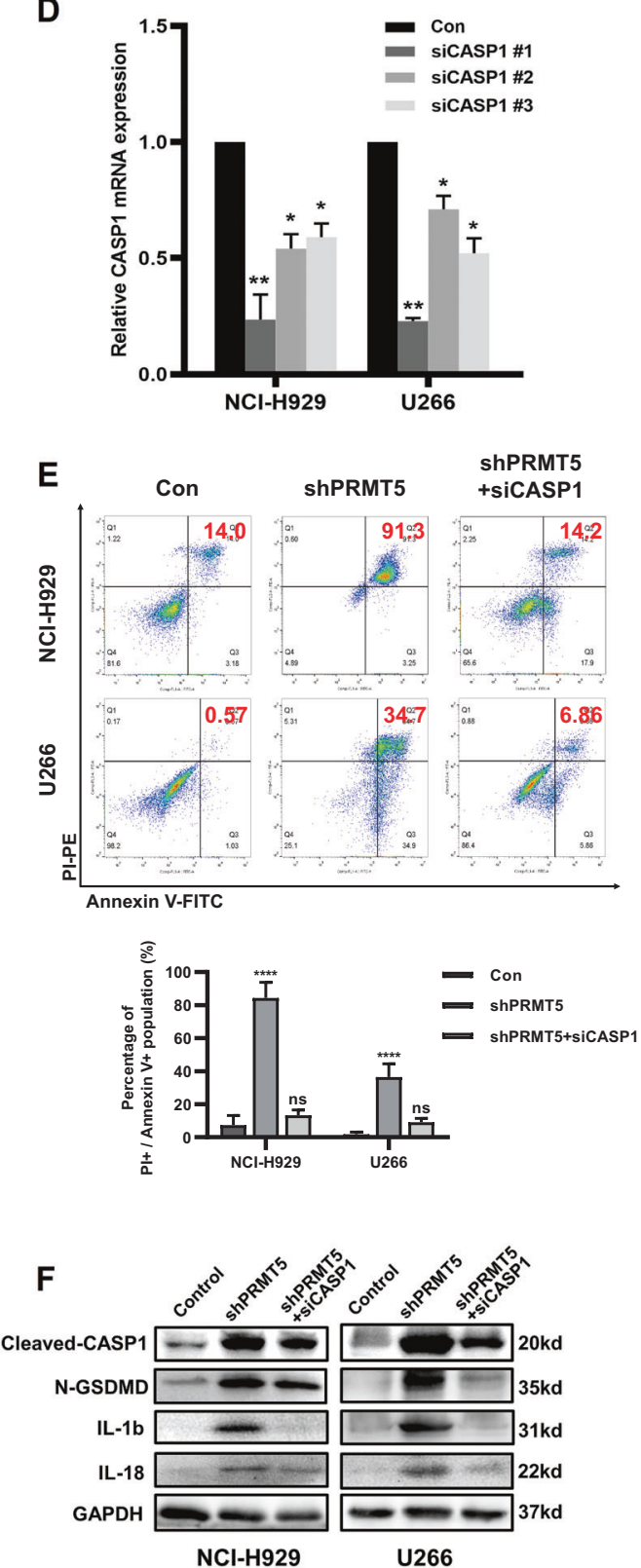

G

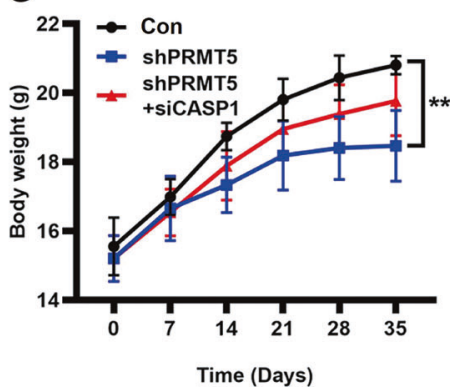

H

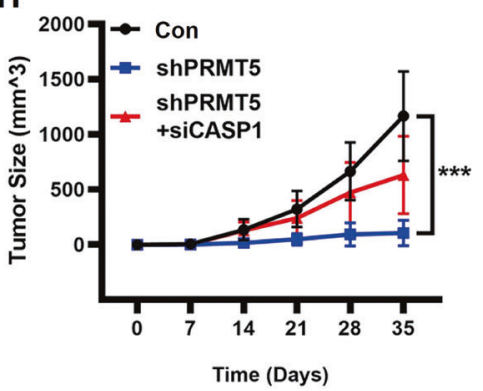

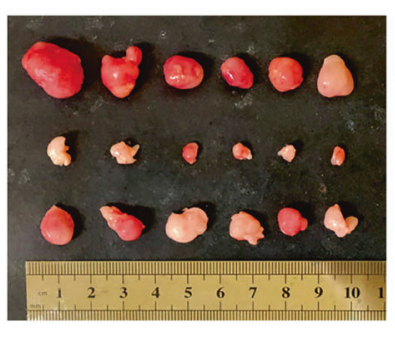

Con

shPRMT5

ShPRMT5

+siCASP1 pathways in vitro, and enhanced tumor progression in vivo. However, these biological phenotypes were inhibited by the knockdown of PRMT5 and then rescued by overexpressing CASP1 in PRMT5-depleted cells, indicating that PRMT5 regulates cell pyroptosis by silencing CASP1 in MM. This study reported the mechanism and significance of the interaction between CASP1 and PRMT5 for the first time and briefly introduced GSK591 treatment as a potential therapeutic strategy for MM.

Arginine methylation plays a crucial role in posttranscriptional modification [16]. PRMT5, which is the predominant type II methyltransferase-targeting arginine methylation, is upregulated in a variety of hematological malignancies and its overexpression 
Fig. 4 Cell pyroptosis induced by depletion of PRMT5 was rescued by knockdown of CASP1. A Relative mRNA expression of CASP1 in NCIH929 cells and U266 cells transfected with the control plasmid (Con) or CASP1 overexpression plasmid (CASP1-OE). B The analysis of Annexin V-FITC and PI by flow cytometry in NCI-H929 (upper panel) and U266 (lower panel) cells transfected with the control or CASP1-OE plasmid. C Western blotting detection of cleaved-CASP1, N-GSDMD, IL-1b, and IL-18 in NCl-H929 and U266 cells transfected with the control or CASP1-OE plasmid. D Relative mRNA expression of CASP1 in NCl-H929 cells and U266 cells transfected with the control plasmid (Con) or CASP1 siRNAs (siCASP1 \#1/\#2/\#3). E The analysis of Annexin V-FITC and PI by flow cytometry in NCI-H929 (upper panel) and U266 (lower panel) cells transfected with PRMT5 shRNA with or without the co-transfected CASP1 siRNAs. F Western blotting detection of Cleaved-CASP1, N-GSDMD, IL-1b, and IL-18 in NCI-H929 and U266 cells transfected with PRMT5 shRNA with or without the co-transfected CASP1 siRNAs. G Weight curves of xenograft mice carrying scr, shPRMT5, or shPRMT5 + siCASP1-transfected NCl-H929 cells. H Images and growth curves of xenograft tumors treated with scr, shPRMT5, or shPRMT5 + siCASP1-transfected NCl-H929 cells. Data shown are mean \pm SD $(n=3)$. ${ }^{*} P<0.05,{ }^{*} P<0.01$, $* * * P<0.001$.

A
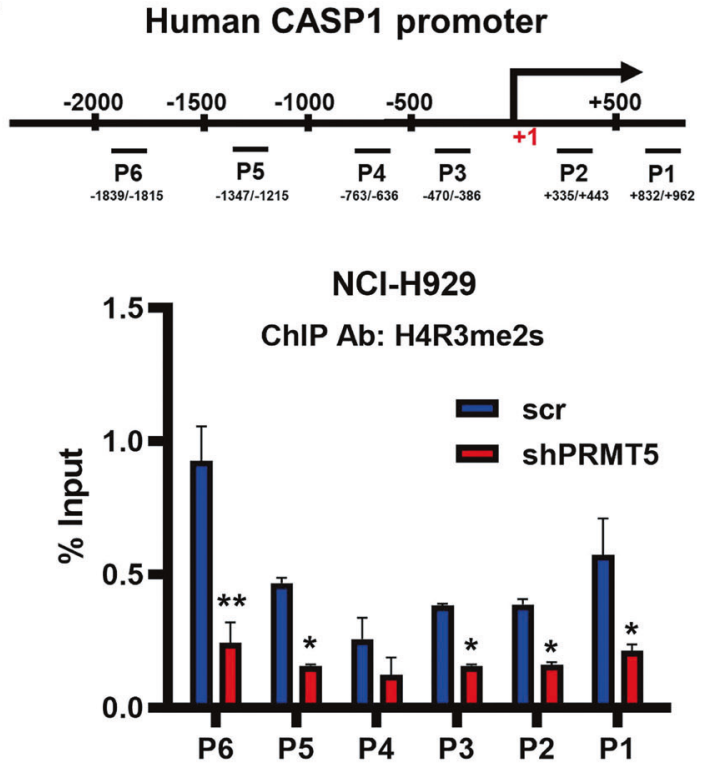

U266

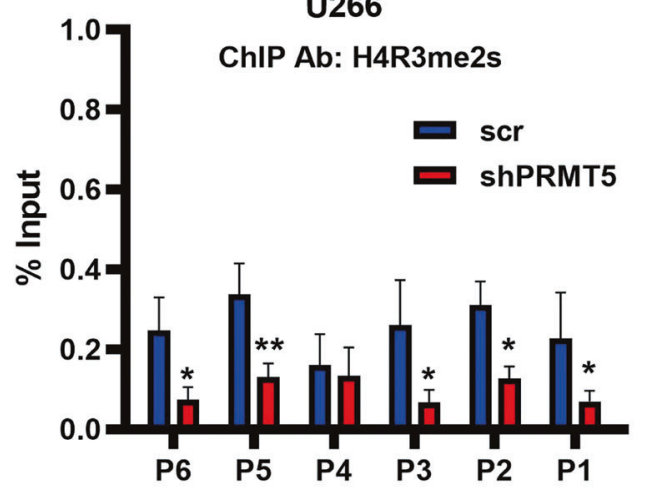

B
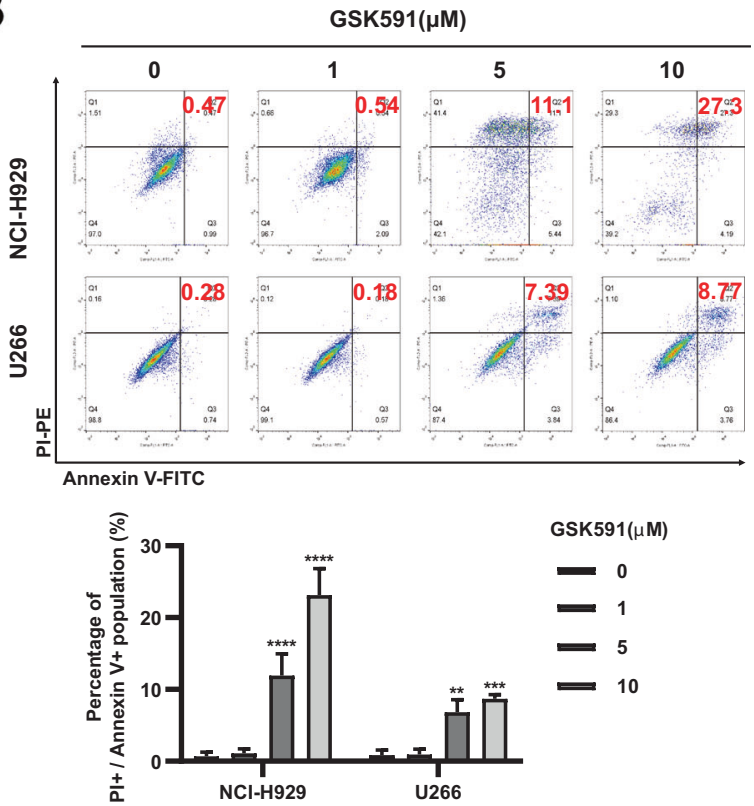

GSK591( $\mu \mathrm{M})$

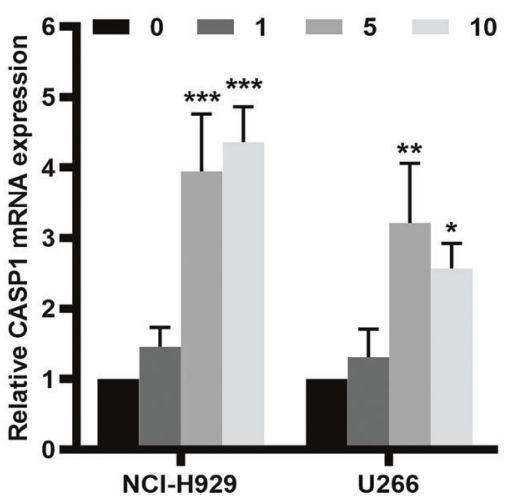

Fig. 5 Treatment of PRMT5 inhibitor GSK591 reduces the level of H4R3me2s at the CASP1 promoter. A Schematic drawing of the CASP1 promoter region. The positions of ChIP-qPCR primer set were labeled relative to the transcriptional start site (TSS). TSS is assigned as the ' +1 ' position. PRMT5-mediated H4R3me2s modifications were significantly less enriched the core promoter regions of CASP1 genes in NCl-H929 cells (upper panel) and U266 cells (lower panel) stably expressing PRMT5 shRNA than scr shRNA by ChIP analysis. IgG was used as a negative control. The bar graph shows percentages of relative fold enrichment of H4R3me2s at different positions across the CASP1 promoter region, compared with the input. B The analysis of Annexin V-FITC and PI by flow cytometry in NCI-H929 (upper panel) and U266 (lower panel) cells treated with $0,1,5,10 \mu \mathrm{M}$ of GSK591. C Relative mRNA expression of CASP1 in NCI-H929 and U266 cells treated with 0, 1, 5, $10 \mu \mathrm{M}$ of GSK591. Data shown are mean \pm SD $(n=3) .{ }^{*} P<0.05,{ }^{* *} P<0.01,{ }^{* * *} P<0.001$.

is associated with tumor aggressiveness and poor overall survival [17-21]. Several therapeutic agents targeting PRMT5 have entered early clinical trials [22]. A study by Ludivine et al. [23] revealed that PRMT5 prevents premature plasma cell differentiation. Gullà et al. [13] found that inhibition of PRMT5 by EPZ015666 inhibits MM cell proliferation and decreases tumor growth through
TRIM21-dependent NF-kB inhibition. PRMT5 inhibition was also reported to reduce the expression of $\mathrm{E} 2 \mathrm{~F}$ targets and alter the methylation status of E2F1 in JAK2V617F-mutant myeloproliferative neoplasms [22]. Several studies have supported the ability of PRMT5 to activate the transcriptional regulation of downstream molecules. A study by Ge et al. [24] revealed that PRMT5 induces 
A

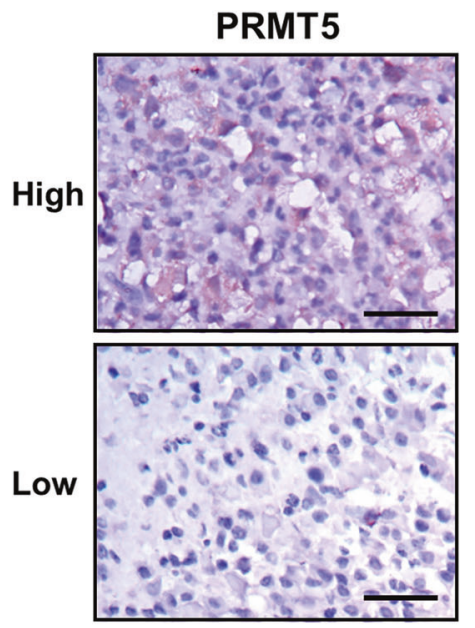

B

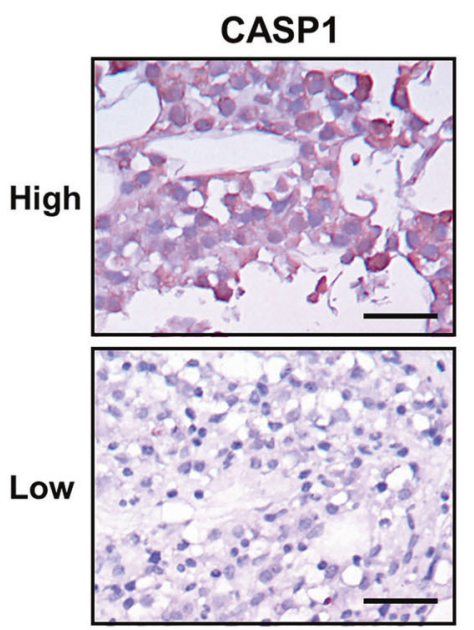

C

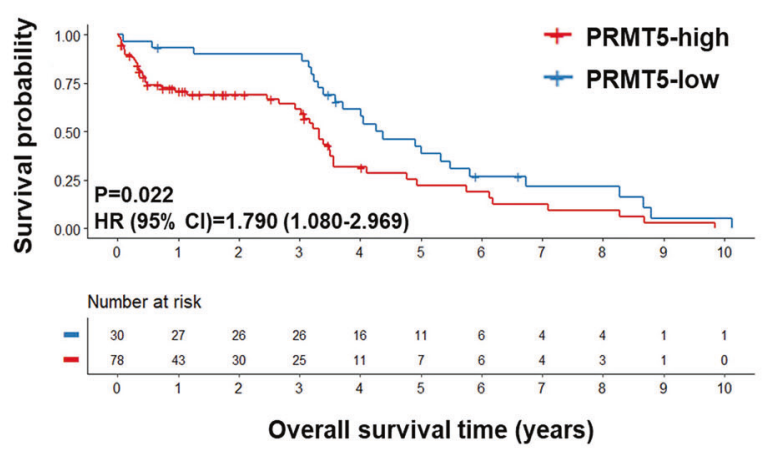

E

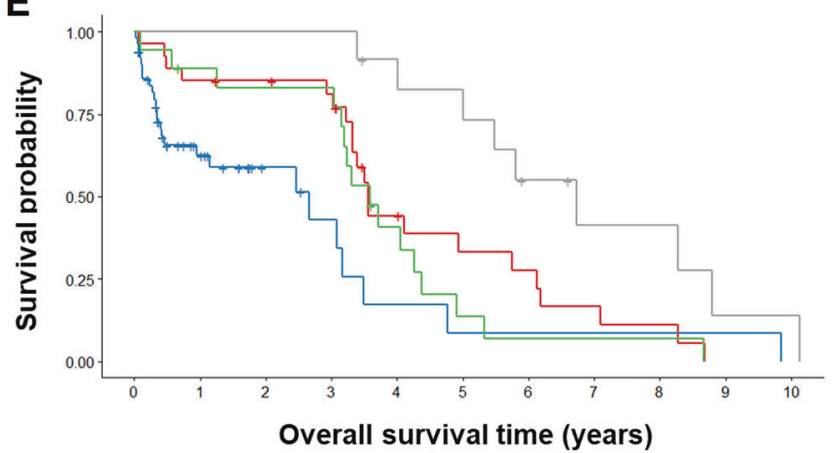

Overall survival time (years)
D

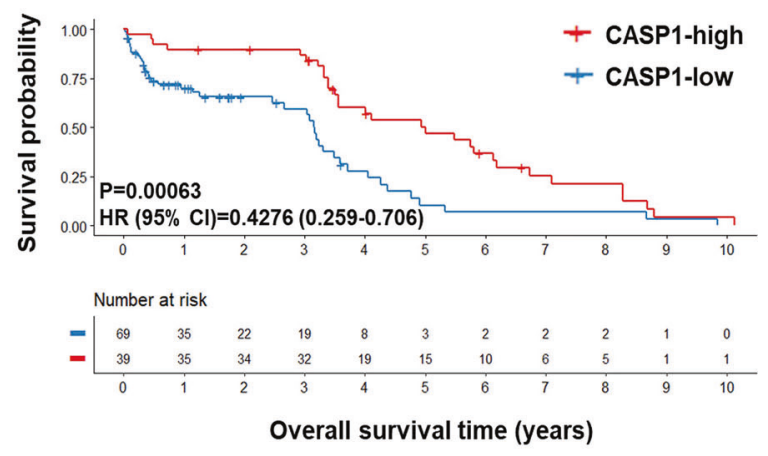

$\mathbf{F}$

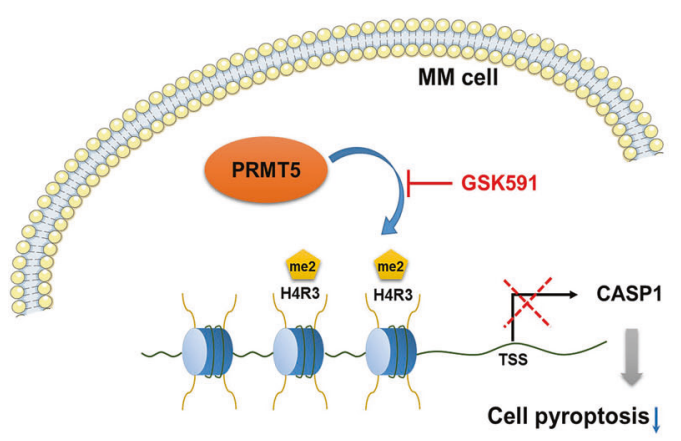

\footnotetext{
+ High PRMT5 + low CASP1

+ High PRMT5 + high CASP1 $(P=0.0415)$

+ Low PRMT5 + highCASP1 (P=0.0037)

+ Low PRMT5 + low CASP1 ( $P=0.1295)$
}

Fig. 6 High expression of PRMT5 and low expression of CASP1 associate with poor clinical outcomes in MM patients. A, B Representative images of IHC staining of PRMT5 and CASP1 in MM bone marrow tissues, which were divided into a high-expression group (upper panel) and a low-expression group (lower panel). Scale bar $=50 \mu \mathrm{m}$. C Overall survival of high PRMT5 $(n=78)$ and low PRMT5 $(n=30)$ MM patients performed by Kaplan-Meier survival analysis. D Overall survival of high CASP1 $(n=39)$ and low CASP1 $(n=69)$ MM patients performed by Kaplan-Meier survival analysis. E Kaplan-Meier survival analysis of MM patients based on both PRMT5 and CASP1 expression. MM patients were divided into four groups: high PRMT5 and low CASP1 expression $(n=51)$, high PRMT5 and high CASP1 expression ( $n=27)$, low PRMT5 and high CASP1 expression $(n=12)$, and low PRMT5 and low CASP1 expression $(n=18)$. P-values were calculated using the group of high PRMT5 and low CASP1 expression as the reference group. F A model for PRMT5-dependent cell proliferation and apoptosis by silencing CASP1 in MM. 
Table 1. The relationship between PRMT5 expression and clinicopathological characteristics in MM.

\begin{tabular}{|c|c|c|c|c|c|}
\hline \multirow[t]{2}{*}{ Characteristics } & \multirow[t]{2}{*}{ No. } & \multicolumn{2}{|c|}{$\begin{array}{l}\text { Expression of PRMT5 } \\
\text { level in MM }\end{array}$} & \multirow[t]{2}{*}{$x^{2}$} & \multirow[t]{2}{*}{$P$-value } \\
\hline & & Low & High & & \\
\hline$N$ & 108 & 30 & 78 & & \\
\hline \multicolumn{6}{|l|}{ Gender } \\
\hline Male & 65 & 18 & 47 & 0.001 & 0.981 \\
\hline Female & 43 & 12 & 31 & & \\
\hline \multicolumn{6}{|l|}{ Age (years) } \\
\hline$\leq 60$ & 59 & 13 & 46 & 2.139 & 0.144 \\
\hline$>60$ & 49 & 17 & 32 & & \\
\hline \multicolumn{6}{|l|}{ ISS stage } \\
\hline 1 & 18 & 8 & 10 & 8.502 & 0.014 \\
\hline II & 40 & 13 & 27 & & \\
\hline III & 50 & 7 & 43 & & \\
\hline \multicolumn{6}{|l|}{ M protein type } \\
\hline IgG type & 50 & 11 & 39 & 5.81 & 0.325 \\
\hline IgA type & 30 & 9 & 21 & & \\
\hline lgM type & 1 & 0 & 1 & & \\
\hline lgD type & 5 & 3 & 2 & & \\
\hline Light chain type & 19 & 7 & 12 & & \\
\hline Non-secretary type & 3 & 0 & 3 & & \\
\hline
\end{tabular}

MM multiple myeloma, ISS international staging system. Bold values are statistically significant $(P<0.05)$.

H4R3 symmetric dimethylation to increase the transcription of FGFR3 and elF4E. Liu et al. [11] demonstrated that PRMT5-mediated H4R3me2s epigenetically repressed transcription of the c-Myc target genes, PTEN, p18, p21, p57, and p63, to promote cell proliferation and gastric cancer progression. Deng et al. [25] revealed that PRMT5 interacts with BRG1 and $\mathrm{Sp} 1$ to induce symmetrical dimethylation modification of H4R3 in the promoter region of the androgen receptor gene, activating androgen receptors and promoting the proliferation of prostate cancer cells. In our study, we explored the CASP1-mediated pyroptosis pathway and demonstrated that PRMT5-induced H4R3me2s silences the transcription of CASP1 to suppress cellular pyroptosis in MM (Fig. 6F).

PRMT5 induces gene silencing not only by generating repressive histone marks such as $\mathrm{H} 4 \mathrm{R} 3 \mathrm{me} 2 \mathrm{~s}$, but also by methylating nonhistone proteins, such as the transcription factors p53 [26, 27], E2F1 $[22,28]$, and p65 $[12,26]$, as well as intercellular protein molecules, such as Sm protein [29], ribosomal protein S10 (RPS10) [30], and rapidly accelerated fibrosarcoma (RAF) [31]. Investigating these interactions of genes with PRMT5 in MM will be of great interest.

The CASP1 gene encodes caspase 1, a member of the cysteineaspartic acid protease (caspase) family which primarily involves the inflammatory process [32]. However, CASP1-mediated cell pyroptosis has also been shown to participate in various tumor development stages [33-35]. CASP1 initiates the pyroptosis pathway through cleavage of GSDMD, and it also proteolytically cleaves the precursors of the inflammatory cytokines IL-1b and IL-18 [36-38] and is essential to distinguish pyroptosis from apoptosis and necrosis, since all of them exhibit an elevated population of PI+ cells by flow cytometry analysis, as shown by our results. Necrosis is a passive and uncontrolled cell death pathway customarily induced by extreme environmental stimuli, such as hypoxia. Intercellular contents overflow to the peripheral environment, causing inflammation and tissue damage [39]. Apoptosis is the process by which a cell ceases to grow and divide, and instead enters a process that ultimately results in the controlled death of the cell without spillage of its contents into the surrounding environment [40]. There are two pathways of apoptosis, intrinsic and extrinsic ways, both of which converge, resulting in cell death when the executioner caspase CASP3 is activated [40-42]. Accordingly, we detected the expression of CASP3 in PRMT5-knockdown and negative control MM cell lines, and the results showed no significance, suggesting only an infinitesimal possibility of apoptosis activation. Here, we demonstrate that CASP1 is a possible target of PRMT5 in MM. However, the coenrichment of CASP1 with H4R3me2s remains unknown and needs further research. Similarly, although our results revealed crosstalk between PRMT5 and the CASP1-mediated pyroptosis pathway, the detailed mechanism remains to be determined.

Our results implied the possibility that the PRMT5 inhibitor GSK591 may represent a therapeutic approach for MM. In fact, PRMT5 inhibitors have shown strong therapeutic potential in oncotherapy, with some of them already commercialized. Traditional inhibitors of PRMT5 mainly consist of SAM analogs (DS-437 [43], MTA [44], etc.), CMP5 [45], and CMP5 derivatives (HLCL-61 [19], EPZ01586 [20], etc.). Novel small-molecule PRMT5 inhibitors (GSK591, GSK3326595, etc.) hinder its methyltransferase activity by suppressing the formation of the PRMT5/MEP50 complex. Scientists from the Sanford Burnham Prebys Medical Discovery Institute found that combined treatment with PRMT5 inhibitors and PD-1 inhibitors successfully enhanced the response to antitumor immunity by regulating methylation of interferon- $\gamma$ (IFN- $\gamma$ )-inducible protein 16 (IFI16) and its murine homolog IFI204, which are components of CGAS/STING, and by inhibiting transcription of the nucleotide-binding oligomerization domainlike receptor family caspase recruitment domain containing 5 (NLRC5) gene in melanoma [46]. At present, several PRMT5 inhibitors are being examined in clinical trials, including GSK3326595 (phase II clinical), JNJ-64619178, and PF-06939999.

We explored the biological function of PRMT5 in MM and then focused on its mechanism of regulating the pyroptosis pathway both in vitro and in vivo. Taken together, our findings demonstrate that PRMT5 regulates cell pyroptosis by silencing CASP1 in MM, and the PRMT5 inhibitor GSK591 may serve as a potential therapeutic agent for MM.

\section{MATERIALS AND METHODS}

\section{Cell lines and cell culture}

MM cell lines (NCl-H929, RPMI-8226, and U266) were purchased from American Type Culture Collection (ATCC). All cells were cultured in RPMI 1640 medium (Gibco, Carlsbad, CA) supplemented with 10\% fetal bovine serum (Gibco) in an atmosphere at $37^{\circ} \mathrm{C}$ with $5 \% \mathrm{CO}_{2}$.

\section{CDNA preparation and qRT-PCR}

Total RNA was extracted using RNA-easy Isolation Reagent (R701-01/02, Vazyme Biotech) and quantified using an ultramicro spectrophotometer (NanoPhotometer-NP80, USA). Reverse transcription was performed using ABScript II RT Mix for qPCR with gDNA Remover (RK20403, Abclonal) according to the manufacturer's protocol. Quantitative real-time PCR analysis was performed using qPCR SYBR Green Master mix (Q311-02, Vazyme Biotech) with an Applied Biosystems ${ }^{\oplus}$ QuantStudio $^{\mathrm{TM}} 6$ Flex RealTime PCR System. The relative quantitative value was calculated using the $2^{-\Delta \Delta C t}$ method. The final result was recorded as fold change. Every sample was run using with three replicates. The primers used for qRT-PCR are described in Supplemental Table 1.

\section{Patient samples and immunohistochemistry}

Bone marrow samples of newly diagnosed MM patients were collected at Nanjing Drum Tower Hospital, Jiangsu Province, China. MM patients with a distinctive pathologic diagnosis, no preoperative systemic or local treatment, and complete follow-up data were selected for inclusion in this study. The paraffin-embedded tissue were deparaffinized, rehydrated, and then subjected to antigen retrieval. The tissue slides were, respectively, incubated with PRMT5 antibodies (1:200; Sigma) and CASP1 
Table 2. Univariate and multivariate analyses of clinicopathological characteristics for survival in patients with MM.

\begin{tabular}{|c|c|c|c|}
\hline \multirow[t]{2}{*}{ Characteristics } & \multirow[t]{2}{*}{ Univariate analysis $P$-value } & \multicolumn{2}{|c|}{ Multivariate analysis } \\
\hline & & $P$-value & HR (95\% Cl) \\
\hline Expression of PRMT5 & $<0.001$ & 0.007 & $3.940(1.454-10.678)$ \\
\hline Age & 0.180 & 0.008 & $0.393(0.197-0.768)$ \\
\hline ISS stage & $<0.001$ & $<0.001$ & $5.091(2.600-9.969)$ \\
\hline M protein type & 0.699 & 0.701 & $0.958(0.768-1.194)$ \\
\hline
\end{tabular}

$H R$ hazard ratio, $\mathrm{Cl}$ confidence interval. Bold values are statistically significant $(P<0.05)$.

antibodies (1:200; CST) overnight at $4{ }^{\circ} \mathrm{C}$. Subsequently, the slides were incubated with horseradish peroxidase (HRP)-conjugated secondary antibody. Each sample was independently scored by two pathologists who were blinded to all the patient clinical data. Based on the percentage of PRMT5- or CASP1-positive tumor cells, the extent of staining was scored 0 (negative), $1(1-25 \%), 2(26-50 \%), 3(51-75 \%)$, or $4(76-100 \%)$. The final score for each slide was assessed by multiplying the scores for intensity and extent of staining. PRMT5 or CASP1 expression on slides was considered low if the score was $<3$ and high if the score was $>3$.

\section{Transfection of cell lines}

PRMT5 shRNA lentivirus was prepared by the co-transfection of 293T cells with pLKO. 1 and the packaging plasmids PMD2G and psPAX2. At $48 \mathrm{~h}$ after transfection, the viral supernatants were collected. NCI-H929 or U266 cells were incubated with viral supernatants in the presence of polybrene. Positive cells were selected using $1 \mu \mathrm{g} / \mathrm{ml}$ puromycin. The target sequences were PRMT5 shRNA\#1: 5'-CCCATCCTCTT CCCTATTAAG-3' and PRMT5 shRNA\#2: 5'-GCCCAGTT TGAGATGCCTTAT-3'. A scrambled sequence was used as a negative control.

CASP1 siRNAs were synthesized by SyngenTech Inc. (Beijing, China) and transfected using Lipofectamine 2000 (Invitrogen, USA) according to the manufacturer's protocol. SiRNA sequences targeting CASP1 were siRNA\#1: 5'-GGUGUGGUUUAAAGAUUCATT-3'; SIRNA\#2: 5'-GAAGACUCAUUGAACAUAUTT-3'; and siRNA\#3: 5'-CUCUCAAGGAGUACUUUCUTT-3'.

CASP1 overexpression plasmids and the control plasmid were purchased from SyngenTech Inc. (Beijing, China) and were transfected into cells using Lipofectamine 2000 (Invitrogen, USA) according to the manufacturer's protocol.

\section{Protein extraction and western blotting}

Cells were washed with PBS and lysed in lysis buffer $(20 \mathrm{mM}$ Tris at pH 7.5, $150 \mathrm{mM} \mathrm{NaCl}, 1 \%$ Triton $\mathrm{X}-100$, sodium pyrophosphate, $\beta$-glycerophosphate, EDTA, $\mathrm{Na}_{3} \mathrm{VO}_{4}$, leupeptin). The concentration of the protein was quantified using the BCA protein assay (Thermo Fisher Scientific, USA). Western blotting was implemented in accordance with the standard methods [47]. The antibodies used were as follows: PRMT5 (P0493, Sigma), GAPDH (HRP-60004, Proteintech), CASP1 (3866, CST), cleaved-CASP1 (4199, CST), H4R3me2s (ab5823, Abcam), cleaved-CASP3 (9661, CST), N-GSDMD (ab215203, Abcam), IL-1b (66737-1-lg, Proteintech), and IL-18 (10663-1-AP, Proteintech). Quantifications of all western blotting signals with 3 repeated experiments are shown in Supplemental Fig. 2A-D as histograms.

\section{GeneChip}

According to the manufacturer's instructions, biotinylated CRNA were prepared according to the standard Affymetrix protocol from $6 \mu \mathrm{g}$ total RNA (Expression Analysis Technical Manual, 2001, Affymetrix). Following fragmentation, $10 \mu \mathrm{g}$ of cRNA was hybridized for $16 \mathrm{~h}$ at $45^{\circ} \mathrm{C}$ on GeneChip Rat Gene 2.0. GeneChip were washed and stained in the Affymetrix Fluidics Station 400. GeneChip were scanned using the Hewlett-Packard GeneArray Scanner G3000 7G. The data were analyzed with RMA using Affymetrix default analysis settings and global scaling as normalization method. The trimmed mean target intensity of each array was arbitrarily set to 100 .

\section{Chromatin immunoprecipitation (ChIP)}

Cells were cultured in RPMI-1640 with $10 \%$ FBS and then cross-linked with $1 \%$ formaldehyde at room temperature. Then, 10-15 min later, the reaction was quenched with glycine for $10 \mathrm{~min}$ at a final concentration of $125 \mathrm{mM}$. Subsequently, the chromatin was sonicated to produce DNA fragments (200-500 bp). For this, $100 \mu \mathrm{g}$ of chromatin was incubated overnight with rotation at $4^{\circ} \mathrm{C}$ with H4R3me2s (Abcam) antibodies or lgG $(2 \mu \mathrm{g})$ followed by $1 \mathrm{~h}$ incubation with protein A Sepharose beads. After washing with lowsalt buffer and high-salt buffer, the beads were incubated with elution buffer and proteinase $\mathrm{K}$ at $65^{\circ} \mathrm{C}$ overnight to immunoprecipitate the DNA. Total DNA fragments were isolated by phenol/chloroform extraction and ethanol precipitation. After isolation, the DNA was diluted in water and subjected to real-time PCR analysis. The primers designed for qRT-PCR are shown in Supplemental Table 2.

\section{Immunofluorescence}

$\mathrm{NCl}-\mathrm{H} 929$ cells and U266 cells were cultured on glass coverslips, fixed in 4\% paraformaldehyde (Sigma) for $30 \mathrm{~min}$ and permeabilized using $0.3 \%$ Triton $\mathrm{X}-100$ for $30 \mathrm{~min}$ at room temperature. After blocking with $5 \%$ goat serum in PBS, cells were incubated with PRMT5 antibodies (Sigma) for $60 \mathrm{~min}$ and secondary antibodies for $45 \mathrm{~min}$, respectively, in a dark and humid chamber. Then, the cells were washed with PBS, and the nuclei were stained with 4,6,diarnidino-2-phenylindole (DAPI) for 5-10 min. Immunofluorescence images were captured under a fluorescence microscope (BX53, Olympus).

\section{CCK-8 assay}

The indicated cells were seeded into each well at a density of 1000 cells in 96-well plates. We examined cell growth every $24 \mathrm{~h}$ using a Cell Counting Kit-8 kit (CCK-8, Dojindo, Japan) after incubation for $1 \mathrm{~h}$ at $37^{\circ} \mathrm{C}$. According to the reference wavelength, the absorbance value was detected at $450 \mathrm{~nm}$.

\section{Flow cytometry}

An Annexin V-FITC/PI kit (BD Biosciences) was used to measure apoptosis according to the manufacturer's protocol. Briefly, $10^{5}$ cells were collected and washed twice with PBS. A total of $50 \mu \mathrm{l}$ binding buffer, $5 \mu \mathrm{l}$ Annexin VFITC, and $5 \mu \mathrm{l} \mathrm{PI}$ were added to the cell suspension and mixed at room temperature in the dark for $10 \mathrm{~min}$. Apoptosis was measured using a flow cytometer (BD Accuri C6 Plus) within $1 \mathrm{~h}$. The flow cytometry data were analyzed using FlowJo 10.

\section{Tumor growth in xenografts}

In all, 18 female BALB/c nude mice were fed sterilized feed and pure water in a specified pathogen-free environment in an air laminar flow chamber. Feed, bedding, and cages for indoor use were autoclaved and transported through a sterile inlet chamber to exclude any microorganisms. Then, the mice were divided into three groups and subcutaneously inoculated with $1 \times 10^{7} \mathrm{NCl}-\mathrm{H} 929$ cells transfected with scr shRNA (Group $\mathrm{A}, n=6$ ), PRMT5 shRNA (Group B, $n=6$ ), or PRMT5 shRNA plus CASP1 siRNA (Group $C, n=6$ ). All mice were weighed, and xenografts were measured using Vernier calipers every week after injection. Five weeks later, all the mice were euthanized by cervical dislocation, and the subcutaneous tumor xenografts of the mice were collected. The maximum diameter of the tumor xenografts was measured using Vernier calipers.

\section{Statistical analysis}

Statistical analysis was performed using Student's $t$-test for comparing two groups using GraphPad Prism software. Data are shown as mean \pm SD. Differences in the mean values were considered to be significant at $P<0.05$. 


\section{DATA AVAILABILITY}

All data included in this study are available from the corresponding authors upon request.

\section{REFERENCES}

1. Kumar SK, Rajkumar V, Kyle RA, van Duin $M$, Sonneveld $P$, Mateos $M V$, et al. Multiple myeloma. Nat Rev Dis Prim. 2017;3:17046.

2. Liu J, Liu W, Mi L, Zeng X, Cai C, Ma J, et al. Incidence and mortality of multiple myeloma in China, 2006-2016: an analysis of the Global Burden of Disease Study 2016. J Hematol Oncol 2019;12:136.

3. Terpos E, Christoulas D, Gavriatopoulou M, Dimopoulos MA. Mechanisms of bone destruction in multiple myeloma. Eur J Cancer Care (Engl). 2017;26:1-11.

4. Dimopoulos MA, Kastritis E, Rosinol L, Blade J, Ludwig H. Pathogenesis and treatment of renal failure in multiple myeloma. Leukemia 2008;22:1485-93.

5. Galm O, Wilop S, Reichelt J, Jost E, Gehbauer G, Herman JG, et al. DNA methylation changes in multiple myeloma. Leukemia 2004;18:1687-92.

6. Galm O, Yoshikawa H, Esteller M, Osieka R, Herman JG. SOCS-1, a negative regulator of cytokine signaling, is frequently silenced by methylation in multiple myeloma. Blood 2003;101:2784-8.

7. Liu H, Liu Z, Du J, He J, Lin P, Amini B, et al. Thymidine phosphorylase exerts complex effects on bone resorption and formation in myeloma. Sci Transl Med. 2016;8:1-11.

8. Alzrigat $M$, Párraga $A$, Jernberg-Wiklund $H$. Epigenetics in multiple myeloma: from mechanisms to therapy. Semin Cancer Biol. 2018;51:101-15.

9. Kryukov GV, Wilson FH, Ruth JR, Paulk J, Tsherniak A, Marlow SE, et al. MTAP deletion confers enhanced dependency on the PRMT5 arginine methyltransferase in cancer cells. Science 2016;351:1214-8.

10. Tan L, Xiao K, Ye $Y$, Liang $H$, Chen $M$, Luo J, et al. High PRMT5 expression is associated with poor overall survival and tumor progression in bladder cancer. Aging (Albany NY). 2020;12:8728-41.

11. Liu M, Yao B, Gui T, Guo C, Wu X, Li J, et al. PRMT5-dependent transcriptional repression of c-Myc target genes promotes gastric cancer progression. Theranostics 2020;10:4437-52.

12. Zhu F, Rui L. PRMT5 in gene regulation and hematologic malignancies. Genes Dis. 2019;6:247-57.

13. Gullà A, Hideshima T, Bianchi G, Fulciniti M, Kemal Samur M, Qi J, et al. Protein arginine methyltransferase 5 has prognostic relevance and is a druggable target in multiple myeloma. Leukemia 2018;32:996-1002.

14. Li Y, Yang Y, Liu X, Long Y, Zheng Y. PRMT5 promotes human lung cancer cell apoptosis via Akt/Gsk3beta signaling induced by resveratrol. Cell Transpl. 2019;28:1664-73.

15. Zhang $S, M a ~ Y, H u ~ X$, Zheng $Y$, Chen X. Targeting PRMT5/Akt signalling axis prevents human lung cancer cell growth. J Cell Mol Med. 2019;23:1333-42.

16. Bedford MT, Clarke SG. Protein arginine methylation in mammals: who, what, and why. Mol Cell. 2009;33:1-13.

17. Liu F, Zhao X, Perna F, Wang L, Koppikar P, Abdel-Wahab O, et al. JAK2V617Fmediated phosphorylation of PRMT5 downregulates its methyltransferase activity and promotes myeloproliferation. Cancer Cell. 2011;19:283-94.

18. Mei M, Zhang R, Zhou ZW, Ying Z, Wang J, Zhang H, et al. PRMT5-mediated H4R3sme2 confers cell differentiation in pediatric B-cell precursor acute lymphoblastic leukemia. Clin Cancer Res. 2019;25:2633-43.

19. Tarighat SS, Santhanam R, Frankhouser D, Radomska HS, Lai H, Anghelina M, et al. The dual epigenetic role of PRMT5 in acute myeloid leukemia: gene activation and repression via histone arginine methylation. Leukemia 2016;30:789-99.

20. Chan-Penebre E, Kuplast KG, Majer CR, Boriack-Sjodin PA, Wigle TJ, Johnston LD, et al. A selective inhibitor of PRMT5 with in vivo and in vitro potency in $\mathrm{MCL}$ models. Nat Chem Biol. 2015;11:432-7.

21. Chung J, Karkhanis V, Baiocchi RA, Sif S. Protein arginine methyltransferase 5 (PRMT5) promotes survival of lymphoma cells via activation of WNT/beta-catenin and AKT/GSK3beta proliferative signaling. J Biol Chem. 2019;294:7692-710.

22. Pastore F, Bhagwat N, Pastore A, Radzisheuskaya A, Karzai A, Krishnan A, et al. PRMT5 inhibition modulates E2F1 methylation and gene-regulatory networks leading to therapeutic efficacy in JAK2(V617F)-mutant MPN. Cancer Discov. 2020;10:1742-57.

23. Litzler LC, Zahn A, Meli AP, Hébert S, Patenaude AM, Methot SP, et al. PRMT5 is essential for B cell development and germinal center dynamics. Nat Commun. 2019;10:22.

24. Ge S, Zhang Q, Chen Y, Tian Y, Yang R, Chen X, et al. Ribavirin inhibits colorectal cancer growth by downregulating PRMT5 expression and H3R8me2s and H4R3me2s accumulation. Toxicol Appl Pharmacol. 2021;415:115450.

25. Deng X, Shao G, Zhang HT, Li C, Zhang D, Cheng L, et al. Protein arginine methyltransferase 5 functions as an epigenetic activator of the androgen receptor to promote prostate cancer cell growth. Oncogene 2017;36:1223-31.

26. Harris DP, Bandyopadhyay S, Maxwell TJ, Willard B, DiCorleto PE. Tumor necrosis factor (TNF)- $a$ induction of CXCL10 in endothelial cells requires protein arginine methyltransferase 5 (PRMT5)-mediated nuclear factor (NF)-KB p65 methylation. J Biol Chem. 2014;289:15328-39.

27. Gerhart SV, Kellner WA, Thompson C, Pappalardi MB, Zhang XP, Montes de Oca R, et al. Activation of the p53-MDM4 regulatory axis defines the anti-tumour response to PRMT5 inhibition through its role in regulating cellular splicing. Sci Rep. 2018;8:9711.

28. Barczak W, Jin L, Carr SM, Munro S, Ward S, Kanapin A, et al. PRMT5 promotes cancer cell migration and invasion through the E2F pathway. Cell Death Dis. 2020;11:572.

29. Inoue M, Okamoto K, Terashima A, Nitta T, Muro R, Negishi-Koga T, et al. Arginine methylation controls the strength of gammac-family cytokine signaling in $\mathrm{T}$ cell maintenance. Nat Immunol. 2018;19:1265-76.

30. Ren J, Wang $Y$, Liang $Y$, Zhang $Y$, Bao $S, X u$ Z. Methylation of ribosomal protein S10 by protein-arginine methyltransferase 5 regulates ribosome biogenesis. J Biol Chem. 2010;285:12695-705.

31. Andreu-Pérez $P$, Esteve-Puig R, de Torre-Minguela C, López-Fauqued M, BechSerra JJ, Tenbaum $S$, et al. Protein arginine methyltransferase 5 regulates ERK1/ 2 signal transduction amplitude and cell fate through CRAF. Sci Signal. 2011;4:58.

32. Siegel RM. Caspases at the crossroads of immune-cell life and death. Nat Rev Immunol. 2006;6:308-17.

33. Huang T, Zhang P, Li W, Zhao T, Zhang Z, Chen S, et al. G9A promotes tumor cell growth and invasion by silencing CASP1 in non-small-cell lung cancer cells. Cell Death Dis. 2017;8:e2726.

34. Li J, Zhang Y, Ruan R, He W, Qian Y. The novel interplay between CD44 standard isoform and the caspase-1/IL1B pathway to induce hepatocellular carcinoma progression. Cell Death Dis. 2020;11:961.

35. Hong W, Gu Y, Guan R, Xie D, Zhou H, Yu M. Pan-cancer analysis of the CASP gene family in relation to survival, tumor-infiltrating immune cells and therapeutic targets. Genomics 2020;112:4304-15.

36. Gaggero A, De Ambrosis A, Mezzanzanica D, Piazza T, Rubartelli A, Figini M, et al. A novel isoform of pro-interleukin-18 expressed in ovarian tumors is resistant to caspase-1 and -4 processing. Oncogene 2004;23:7552-60.

37. Thornberry NA, Bull HG, Calaycay JR, Chapman KT, Howard AD, Kostura MJ, et al. A novel heterodimeric cysteine protease is required for interleukin-1 beta processing in monocytes. Nature 1992;356:768-74.

38. Shi J, Zhao Y, Wang K, Shi X, Wang Y, Huang H, et al. Cleavage of GSDMD by inflammatory caspases determines pyroptotic cell death. Nature 2015;526:660-5.

39. Elmore S. Apoptosis: a review of programmed cell death. Toxicol Pathol. 2007;35:495-516.

40. D'Arcy MS. Cell death: a review of the major forms of apoptosis, necrosis and autophagy. Cell Biol Int. 2019;43:582-92.

41. Cain K, Bratton SB, Cohen GM. The Apaf-1 apoptosome: a large caspaseactivating complex. Biochimie 2002;84:203-14.

42. Kim JH, Lee SY, Oh SY, Han SI, Park HG, Yoo MA, et al. Methyl jasmonate induces apoptosis through induction of Bax/Bcl-XS and activation of caspase-3 via ROS production in A549 cells. Oncol Rep. 2004;12:1233-8.

43. Smil D, Eram MS, Li F, Kennedy S, Szewczyk MM, Brown PJ, et al. Discovery of a Dual PRMT5-PRMT7 Inhibitor. ACS Med Chem Lett. 2015;6:408-12.

44. Marjon K, Cameron MJ, Quang P, Clasquin MF, Mandley E, Kunii K, et al. MTAP deletions in cancer create vulnerability to targeting of the MAT2A/PRMT5/RIOK1 axis. Cell Rep. 2016;15:574-87.

45. Alinari L, Mahasenan KV, Yan F, Karkhanis V, Chung JH, Smith EM, et al. Selective inhibition of protein arginine methyltransferase 5 blocks initiation and maintenance of B-cell transformation. Blood 2015;125:2530-43.

46. Kim H, Kim H, Feng Y, Li Y, Tamiya H, Tocci S, et al. PRMT5 control of CGAS/STING and NLRC5 pathways defines melanoma response to antitumor immunity. Sci Transl Med. 2020;12:eaaz5683.

47. Burnette WN. "Western blotting": electrophoretic transfer of proteins from sodium dodecyl sulfate-polyacrylamide gels to unmodified nitrocellulose and radiographic detection with antibody and radioiodinated protein A. Anal Biochem. 1981;112:195-203.

\section{ACKNOWLEDGEMENTS}

We would like to thank all the volunteers who took part in this study. This work was supported by the National Natural Science Foundation of China (2020YFA0908204), the Six Talent Peaks Project of Jiangsu Province (2018-WSN-136), and the Jiangsu Provincial Medical Youth Talent and the Technique Development Foundation of Nan Jing (ZKX20014). 


\section{AUTHOR CONTRIBUTIONS}

P.X. and B.C. designed the experiments. J.O. modified experiment plans and provided MM cell lines. P.X. and T.X. performed the experiments. T.X. performed the bioinformatics analysis and data analysis. M.L. and Q.Z. provided experimental guidance and reagents sponsorship. P.X. and T.X. wrote the manuscript together.

\section{COMPETING INTERESTS}

The authors declare no competing interests.

\section{ETHICS APPROVAL AND CONSENT TO PARTICIPATE}

The Ethical Committee of Nanjing Drum Tower Hospital Hematology Center provided permission to use the clinical samples. All patients provided written informed consent for the clinical samples and data to be used for scientific research. The animal experiment was approved by the Animal Ethical and Welfare Committee of Nanjing Drum Tower Hospital and abided by the Guide for the Care and Use of Laboratory Animals.

\section{ADDITIONAL INFORMATION}

Supplementary information The online version contains supplementary material available at https://doi.org/10.1038/s41419-021-04125-5.

Correspondence and requests for materials should be addressed to Jian Ouyang, Peipei Xu or Bing Chen.
Reprints and permission information is available at http://www.nature.com/ reprints

Publisher's note Springer Nature remains neutral with regard to jurisdictional claims in published maps and institutional affiliations. c) Attribution 4.0 International License, which permits use, sharing, adaptation, distribution and reproduction in any medium or format, as long as you give appropriate credit to the original author(s) and the source, provide a link to the Creative Commons license, and indicate if changes were made. The images or other third party material in this article are included in the article's Creative Commons license, unless indicated otherwise in a credit line to the material. If material is not included in the article's Creative Commons license and your intended use is not permitted by statutory regulation or exceeds the permitted use, you will need to obtain permission directly from the copyright holder. To view a copy of this license, visit http://creativecommons. org/licenses/by/4.0/.

(c) The Author(s) 2021, corrected publication 2021 